\title{
Intelligent and pervasive computing for cyber-physical systems
}

\author{
Mohammad R. Khosravi ${ }^{1,2} \cdot$ Varun G. Menon ${ }^{3}$ \\ Published online: 20 October 2020 \\ (c) Springer Science+Business Media, LLC, part of Springer Nature 2020
}

We are happy to introduce the topical collection on "Intelligent and Pervasive Computing for Cyber-Physical Systems" for The Journal of Supercomputing. The subject of cyber-physical systems (CPS) aims to integrate computation, networking and physical processes in Industry 4.0. In these systems, embedded computers for networked monitoring through sensors and control through actuators can help us to manage the physical processes with the use of ultra-reliable and low-latency communications and high-performance computing provided by joint computingnetworking infrastructures in distributed nodes. CPS concerns can also include the related topics in Internet of things (IoT) and distributed sensor networks (DSNs) toward pervasive communications and mobile intelligent computing for the research and development of cutting-edge and advanced applications in smart cities and industrial manufacturing. In recent years, high-performance computing for CPS could achieve much attention among academic researchers and industrial developers. With the rapid developments in artificial intelligence, bio-/nature-inspired soft computing and cyber-physical ubiquitous computing strategies, CPS needs to use them extensively.

This topical collection has tried to highlight the latest research findings and advances focused on how intelligent and pervasive computing is efficiently used in CPS, IoT and DSN, with combining sensory data, control of actuators, and networking and security requirements. The detailed topics covered by the collection are distributed computing techniques for CPS/IoT/DSN, cloud/fog/edge computing techniques and architectures for CPS and IoT, deep learning, neural networks

Mohammad R. Khosravi

mohammadkhosravi@acm.org

Varun G. Menon

varunmenon@ieee.org

1 Department of Computer Engineering, Persian Gulf University, Bushehr, Iran

2 Department of Electrical and Electronic Engineering, Shiraz University of Technology, Shiraz, Iran

3 Department of Computer Science and Engineering, SCMS School of Engineering and Technology, Kochi, India 
and statistical machine learning for CPS and IoT, human-computer interaction and human-in-the-loop for CPS, intelligent sensing techniques and physical processes, security and privacy for CPS/IoT/DSN, protocols and infrastructures for distributed networks, optimization in cyber-physical systems, intelligent multimedia computing in CPS/IoT/DSN, cyber-physical hybrid intelligence and artificial intelligence of things (AIoT), hardware implementation of CPS, and smart energy systems.

This is a pleasure that we have received a large number of submissions from the researchers worldwide. The received manuscripts were reviewed by at least two expert reviewers and one editor such that twenty-five papers were eventually accepted after a rigorous peer review process. Among the accepted papers, most have a great focus on theories and applications behind CPS and two cases are categorized as pure machine learning approaches for general applications [1, 2]. The respected readers are warmly invited to access the collection's papers through [3] and enjoy the content.

At the end, the collection editors would like to sincerely thank the Editor-inChief, Prof. Hamid R. Arabnia, for his kindness and constant support in the time we were handling all the submissions. We also thank the journal admin, Ms. Vinubala Viswanathan, for her valuable assistance and all the contributing authors for their participation.

\section{References}

1. Deng X, Xu Y, Chen L et al (2020) Dynamic clustering method for imbalanced learning based on AdaBoost. J Supercomput 76:9716-9738. https://doi.org/10.1007/s11227-020-03211-3

2. Tavallali P, Tavallali P, Singhal M (2020) K-means tree: an optimal clustering tree for unsupervised learning. J Supercomput. https://doi.org/10.1007/s11227-020-03436-2

3. Khosravi M et al (2020) The topical collection on intelligent and pervasive computing for cyberphysical systems. J Supercomput. Access through: https://link.springer.com/journal/11227/topic alCollection/AC_139af0dd61f6c80979c8197ad5303db8/

Publisher's Note Springer Nature remains neutral with regard to jurisdictional claims in published maps and institutional affiliations. 